\title{
On Recovering Differential Operators on a Closed Set from Spectra
}

\begin{abstract}
V. A. Yurko
Vjacheslav A. Yurko, https://orcid.org/0000-0002-4853-0102, Saratov State University, 83 Astrakhanskaya St., Saratov 410012, Russia, YurkoVA@info.sgu.ru

The Sturm-Liouville differential operators on closed sets of the real line are considered. Properties of their spectral characteristics are obtained and the inverse problem of recovering the operators from their spectra is studied. An algorithm for the solution of the inverse problem is developed and the uniqueness of the solution is established. The statement and the study of inverse spectral problems essentially depend on the structure of the closed set. We consider an important subclass of closed sets when the set is a unification of a finite number of closed intervals and isolated points. In order to solve the inverse spectral problem for this class of closed sets, we develop ideas of the method of spectral mappings. We also establish and use connections between the Weyl-type functions related to different subsets of the main closed set. Using these ideas and properties we obtain a global constructive procedure for the solution of the nonlinear inverse problem considered, and we establish the uniqueness of the solution of the inverse problem.
\end{abstract}

Keywords: differential operators, closed sets, inverse spectral problem.

Received: 01.03.2019 / Accepted: 28.04.2019 / Published: 02.12.2019

This is an open access article distributed under the terms of Creative Commons Attribution License (CC-BY 4.0)

DOI: https://doi.org/10.18500/1816-9791-2019-19-4-389-396

\section{INTRODUCTION}

We study inverse spectral problems for Sturm-Liouville differential operators on a closed set of the real line (in literature it is sometimes called a time scale). Such problems often appear in natural sciences and engineering (see monographs [1,2]).

Inverse spectral problems consist in constructing operators with given spectral characteristics. For the classical differential operators on an interval inverse problems have been studied fairly completely; the main results can be found in [3-15]. However, differential operators defined on closed sets are essentially more difficult for investigating, and nowadays there is no inverse problem theory for this class of operators.

The statement and the study of inverse spectral problems essentially depend on the structure of the closed set. In this paper we will study inverse problems for an important subclass of closed sets. We establish properties of spectral characteristics of Sturm Liouville operators on closed sets and study the inverse problem of recovering the potential of the Sturm - Liouville operator from the given two spectra. The main results of the paper are Theorem 1 and Algorithm 1, where a global constructive procedure for solving the inverse problem is provided, and the uniqueness of the solution is proved.

Let us recall some notions of the time scale theory; see $[1,2]$ for more details. Let $T$ be a closed subset of the real line; it is called sometimes the time scale. We define the so-called jump functions $\sigma$ and $\sigma_{0}$ on $T$ as follows:

$\sigma(x)=\inf \{s \in T: s>x\}$ for $x \neq \sup T$, and $\sigma(\sup T)=\sup T$;

$\sigma_{0}(x)=\sup \{s \in T: s<x\}$ for $x \neq \inf T$, and $\sigma_{0}(\inf T)=\inf T$.

A point $x \in T$ is called left-dense, left-isolated, right-dense and right-isolated, if $\sigma_{0}(x)=x, \sigma_{0}(x)<x, \sigma(x)=x$ and $\sigma(x)>x$, respectively. If $\sigma_{0}(x)<x<\sigma(x)$, then $x$ 
is called isolated; if $\sigma_{0}(x)=x=\sigma(x)$, then $x$ is called dense. A function $f$ on $T$ is called T-continuous, if it is continuous at all right-dense points and has left-sided limits at all left-dense points in $T$. The set of T-continuous functions is denoted by $C_{T}$. Put $T^{0}:=T \backslash\{\sup T\}$, if $\sup T$ is left-isolated, and $T^{0}:=T$, otherwise.

A function $f$ on $T$ is called delta-differentiable at $x \in T^{0}$, if for any $\varepsilon>0$ there exists a neighborhood $U=(x-\delta, x+\delta) \cap T$ such that

$$
\left|(f(\sigma(x))-f(s))-f^{\Delta}(x)(\sigma(x)-s)\right| \leqslant \varepsilon|\sigma(x)-s|
$$

for all $s \in U$. We will call $f^{\Delta}(x)$ the delta-derivative of $f$ at $x$.

Example 1. If $x$ is a right-isolated point, then

$$
f^{\Delta}(x)=\frac{f(\sigma(x))-f(x)}{\sigma(x)-x} .
$$

In particular, if $T=\{x=h k: k \in \mathbf{Z}\}$, then

$$
f^{\Delta}(x)=\frac{f(x+h)-f(x)}{h} .
$$

Example 2. If $x \in T$ is a right-dense point, and $f$ is a delta-differentiable at $x$, then

$$
f^{\Delta}(x)=\lim _{s \rightarrow x, s>x} \frac{f(x)-f(s)}{x-s} .
$$

In particular, if $x \in T$ is a dense point, and $f$ is a delta-differentiable at $x$, then $f$ is differentiable at $x$, and $f^{\Delta}(x)=f^{\prime}(x)$.

\section{DIFFERENTIAL EQUATIONS ON CLOSED SETS}

Consider the Sturm - Liouville equation on $T$ :

$$
-y^{\Delta \Delta}(x)+q(x) y(\sigma(x))=\lambda y(\sigma(x)), \quad x \in T .
$$

Here $\lambda$ is the spectral parameter, $q(x) \in C_{T}$ is a complex-valued function. A function $y$ is called a solution of equation (1), if $y \in C_{T}^{2}$ and satisfies equation (1). The statement and the study of inverse spectral problems essentially depend on the structure of the time scale $T$. It is necessary to choose and describe subclasses of time scales for which the inverse problem theory can be constructed adequately. In this paper we consider one of such subclasses, namely, the so-called $Y 1$-structure. More precisely, we consider the time scale of the form

$$
\begin{gathered}
T=\bigcup_{k=1}^{N}\left[a_{k}, b_{k}\right], \quad N \geqslant 2, \\
b_{k-1}<a_{k} \leqslant b_{k}<a_{k+1}, \quad a_{1}<b_{1}, \quad a_{N}<b_{N}, \quad a_{k}=b_{k}, \quad k=\overline{2, N-1 .} .
\end{gathered}
$$

For $Y 1$-structure one has

$$
y^{\Delta}\left(b_{k}\right)=\frac{y\left(a_{k+1}\right)-y\left(b_{k}\right)}{a_{k+1}-b_{k}}, \quad k=\overline{1, N-1}, \quad y^{\Delta}(x)=y^{\prime}(x), \quad x \in\left[a_{1}, b_{1}\right] \cup\left[a_{N}, b_{N}\right] .
$$

In particular, this yields $y^{\Delta}\left(b_{1}\right)=y^{\prime}\left(b_{1}\right)$, and consequently,

$$
y\left(a_{2}\right)=y\left(b_{1}\right)+\left(a_{2}-b_{1}\right) y^{\prime}\left(b_{1}\right) .
$$


Using (1) and (2) we obtain

$$
\begin{gathered}
-y^{\prime \prime}(x)+q(x) y(x)=\lambda y(x), \quad x \in\left[a_{1}, b_{1}\right] \cup\left[a_{N}, b_{N}\right] \\
y^{\Delta \Delta}\left(b_{k}\right)=\frac{1}{a_{k+1}-b_{k}}\left(\frac{y\left(a_{k+2}\right)-y\left(b_{k+1}\right)}{a_{k+2}-b_{k+1}}-\frac{y\left(a_{k+1}\right)-y\left(b_{k}\right)}{a_{k+1}-b_{k}}\right)= \\
=\left(q\left(b_{k}\right)-\lambda\right) y\left(a_{k+1}\right), \quad k=\frac{1, N-2}{1,} \\
y^{\Delta \Delta}\left(b_{N-1}\right)=\frac{1}{a_{N}-b_{N-1}}\left(y^{\prime}\left(a_{N}\right)-\frac{y\left(a_{N}\right)-y\left(b_{N-1}\right)}{a_{N}-b_{N-1}}\right)=\left(q\left(b_{N-1}\right)-\lambda\right) y\left(a_{N}\right) .
\end{gathered}
$$

Therefore

$$
\begin{gathered}
y\left(a_{k+2}\right)=y\left(b_{k+1}\right)+\frac{a_{k+2}-b_{k+1}}{a_{k+1}-b_{k}}\left(y\left(a_{k+1}\right)-y\left(b_{k}\right)\right)+ \\
+\left(a_{k+1}-b_{k}\right)\left(a_{k+2}-b_{k+1}\right)\left(q\left(b_{k}\right)-\lambda\right) y\left(a_{k+1}\right), \quad k=\overline{1, N-2}, \\
y^{\prime}\left(a_{N}\right)=\frac{y\left(a_{N}\right)-y\left(b_{N-1}\right)}{a_{N}-b_{N-1}}+\left(a_{N}-b_{N-1}\right)\left(q\left(b_{N-1}\right)-\lambda\right) y\left(a_{N}\right) .
\end{gathered}
$$

Let $\lambda=\rho^{2}$. It follows from (3) and (5)-(6) that

$$
y\left(a_{N}\right)=\alpha_{11}(\rho) y\left(b_{1}\right)+\alpha_{12}(\rho) y^{\prime}\left(b_{1}\right), \quad y^{\prime}\left(a_{N}\right)=\alpha_{21}(\rho) y\left(b_{1}\right)+\alpha_{22}(\rho) y^{\prime}\left(b_{1}\right),
$$

where $\alpha_{j k}(\rho)$ are polynomials with respect to $\rho$ of degree $2(N+j-3)$, and they depend on $q\left(b_{1}\right), \ldots, q\left(b_{N+j-3}\right)$. Moreover,

$$
\alpha_{j k}(\rho)=(i \rho)^{2(N+j-3)} \alpha_{j k}^{0}[1], \quad|\rho| \rightarrow \infty,
$$

where $[1]=1+O\left(\rho^{-1}\right)$,

$$
\begin{gathered}
\alpha_{12}^{0}=\left(a_{2}-b_{1}\right) \alpha_{11}^{0}, \quad \alpha_{21}^{0}=\left(a_{N}-b_{N-1}\right) \alpha_{11}^{0}, \quad \alpha_{22}^{0}=\left(a_{2}-b_{1}\right)\left(a_{N}-b_{N-1}\right) \alpha_{11}^{0}, \\
\alpha_{11}^{0}=\left(a_{2}-b_{1}\right)\left(a_{N}-b_{N-1}\right) \prod_{k=2}^{N-2}\left(a_{k+1}-b_{k}\right)^{2}
\end{gathered}
$$

$\left(\alpha_{11}^{0}=1\right.$ for $N=2$, and $\alpha_{11}^{0}=\left(a_{2}-b_{1}\right)\left(a_{3}-b_{2}\right)$ for $\left.N=3\right)$. Without loss of generality we assume that $a_{1}=0$.

Denote by $L_{0}=L_{0}(q)$ the boundary value problem for equation (1) on $T$ with the boundary conditions $y(0)=y\left(b_{N}\right)=0$. Let $S(x, \lambda)$ and $C(x, \lambda)$ be solutions of equation (1) on $T$ satisfying the initial conditions

$$
C(0, \lambda)=S^{\Delta}(0, \lambda)=1, \quad S(0, \lambda)=C^{\Delta}(0, \lambda)=0 .
$$

For each fixed $x$, the functions $S(x, \lambda)$ and $C(x, \lambda)$ are entire in $\lambda$ of order $1 / 2$. Denote

$$
\Delta_{0}(\lambda):=S\left(b_{N}, \lambda\right)
$$

The eigenvalues $\left\{\lambda_{n 0}\right\}_{n \geqslant 1}$ of the boundary value problem $L_{0}$ coincide with the zeros of the entire function $\Delta_{0}(\lambda)$. The function $\Delta_{0}(\lambda)$ is called the characteristic function for $L_{0}$. 
Let $\Phi(x, \lambda)$ be the solution of equation (1) on $T$ satisfying the boundary conditions

$$
\Phi(0, \lambda)=1, \quad \Phi\left(b_{N}, \lambda\right)=0 .
$$

Put $M(\lambda):=\Phi^{\Delta}(0, \lambda)$. The function $M(\lambda)$ is called the Weyl-type function or simply Weyl function. Clearly,

$$
\begin{gathered}
\Phi(x, \lambda)=C(x, \lambda)+M(\lambda) S(x, \lambda), \\
M(\lambda)=-\Delta_{1}(\lambda) / \Delta_{0}(\lambda),
\end{gathered}
$$

where $\Delta_{1}(\lambda):=C\left(b_{N}, \lambda\right)$ is the characteristic function for the boundary value problem $L_{1}=L_{1}(q)$ for equation (1) on $T$ with the boundary conditions $y^{\Delta}(0)=y\left(b_{N}\right)=0$. The zeros $\left\{\lambda_{n 1}\right\}_{n \geqslant 1}$ of $\Delta_{1}(\lambda)$ coincide with the eigenvalues of $L_{1}$.

By the well-known arguments (see, for example [5] we obtain the following fact.

Lemma 1. The specification of the spectra $\left\{\lambda_{n j}\right\}_{n \geqslant 0}, j=0,1$, uniquely determines the characteristic functions via

$$
\Delta_{j}(\lambda)=\prod_{n=0}^{\infty} \frac{\lambda_{n j}-\lambda}{\lambda_{n j}^{0}},
$$

where $\left\{\lambda_{n j}^{0}\right\}_{n \geqslant 0}$ are eigenvalues of the boundary value problems $L_{j}^{0}:=L_{j}(0)$ with the zero potential (the case with a zero eigenvalue requires a small modification).

Now we need to study the asymptotical behavior of the solutions $\Phi(x, \lambda)$ and $S(x, \lambda)$. For this purpose we extent the function $q(x)$ on the whole segment $\left[a_{1}, b_{N}\right]$ such that $q(x) \in C\left[a_{1}, b_{N}\right]$ and arbitrary in the rest. Consider the Sturm - Liouville equation

$$
-y^{\prime \prime}(x)+q(x) y(x)=\lambda y(x), \quad x \in\left[0, b_{N}\right] .
$$

It is known (see, for example, [5]) that there exists a fundamental system of solutions of equation (13) $\left\{Y_{1}(x, \rho), Y_{2}(x, \rho)\right\}, x \in\left[0, b_{N}\right], \operatorname{Im} \rho \geqslant 0,|\rho|>\rho_{0}$, having the following asymptotical behavior for each fixed $x \in\left[0, b_{N}\right]$ as $|\rho| \rightarrow \infty$ :

$$
Y_{1}^{(\nu)}(x, \rho)=(i \rho)^{\nu} \exp (i \rho x)[1], \quad Y_{2}^{(\nu)}(x, \rho)=(-i \rho)^{\nu} \exp (-i \rho x)[1], \quad \nu=0,1 .
$$

The function $\Phi(x, \lambda)$ is the solution of equation (4) satisfying the boundary conditions (9) and the jump conditions (7), i.e.

$$
\begin{gathered}
\Phi\left(a_{N}, \lambda\right)=\alpha_{11}(\rho) \Phi\left(b_{1}, \lambda\right)+\alpha_{12}(\rho) \Phi^{\prime}\left(b_{1}, \lambda\right), \\
\Phi^{\prime}\left(a_{N}, \lambda\right)=\alpha_{21}(\rho) \Phi\left(b_{1}, \lambda\right)+\alpha_{22}(\rho) \Phi^{\prime}\left(b_{1}, \lambda\right) .
\end{gathered}
$$

Using the fundamental system of solutions $\left\{Y_{1}(x, \rho), Y_{2}(x, \rho)\right\}$, one has

$$
\begin{gathered}
\Phi(x, \lambda)=A_{1}(\rho) Y_{1}(x, \rho)+A_{2}(\rho) Y_{2}(x, \rho), \quad x \in\left[0, b_{1}\right], \\
\Phi(x, \lambda)=B_{1}(\rho) Y_{1}(x, \rho)+B_{2}(\rho) Y_{2}(x, \rho), \quad x \in\left[a_{N}, b_{N}\right] .
\end{gathered}
$$

Substituting (16) into (9) and (15) and using (14), we obtain the following linear algebraic system with respect to $A_{k}(\rho)$ and $B_{k}(\rho)$ :

$$
A_{1}(\rho)[1]+A_{2}(\rho)[1]=1, \quad B_{1}(\rho) \exp \left(i \rho b_{N}\right)[1]+B_{2}(\rho) \exp \left(-i \rho b_{N}\right)[1]=0,
$$




$$
\begin{gathered}
B_{1}(\rho) \exp \left(i \rho a_{N}\right)[1]+B_{2}(\rho) \exp \left(-i \rho a_{N}\right)[1]= \\
=\alpha_{11}(\rho)\left(A_{1}(\rho) \exp \left(i \rho b_{1}\right)[1]+A_{2}(\rho) \exp \left(-i \rho b_{1}\right)[1]\right)+ \\
+\alpha_{12}(\rho)\left(A_{1}(\rho)(i \rho) \exp \left(i \rho b_{1}\right)[1]+A_{2}(\rho)(-i \rho) \exp \left(-i \rho b_{1}\right)[1]\right), \\
B_{1}(\rho)(i \rho) \exp \left(i \rho a_{N}\right)[1]+B_{2}(\rho)(-i \rho) \exp \left(-i \rho a_{N}\right)[1]= \\
=\alpha_{21}(\rho)\left(A_{1}(\rho) \exp \left(i \rho b_{1}\right)[1]+A_{2}(\rho) \exp \left(-i \rho b_{1}\right)[1]\right)+ \\
+\alpha_{22}(\rho)\left(A_{1}(\rho)(i \rho) \exp \left(i \rho b_{1}\right)[1]+A_{2}(\rho)(-i \rho) \exp \left(-i \rho b_{1}\right)[1]\right) .
\end{gathered}
$$
form

Taking (8) into account we deduce that the determinant $D(\rho)$ of this system has the

$$
\begin{gathered}
D(\rho)=(i \rho) \alpha_{22}(\rho)\left(\exp \left(i \rho\left(b_{N}-a_{N}\right)\right)[1]-\exp \left(-i \rho\left(b_{N}-a_{N}\right)\right)[1]\right) \times \\
\times\left(\exp \left(i \rho\left(b_{1}-a_{1}\right)\right)[1]-\exp \left(-i \rho\left(b_{1}-a_{1}\right)\right)[1]\right), \quad|\rho| \rightarrow \infty, \quad \operatorname{Im} \rho \geqslant 0 .
\end{gathered}
$$

Denote $\Omega_{\delta}:=\{\rho: \arg \rho \in[\delta, \pi-\delta]\}$. Solving this algebraic system by Cramer's rule and using (17), we get

$$
\begin{gathered}
A_{1}(\rho)=[1], \quad A_{2}(\rho)=\exp \left(2 i \rho b_{1}\right)[1] \\
B_{1}(\rho)=\exp \left(-i \rho\left(a_{N}-b_{1}\right)\right) O\left(\rho^{2 N-4}\right)[1] \\
B_{2}(\rho)=\exp \left(-i \rho\left(a_{N}-b_{1}\right)\right) \exp \left(2 i \rho b_{N}\right) O\left(\rho^{2 N-4}\right)[1] .
\end{gathered}
$$

for $|\rho| \rightarrow \infty, \rho \in \Omega_{\delta}$.

In particular this yields for each fixed $x \in\left[0, b_{1}\right)$ :

$$
\Phi^{(\nu)}(x, \lambda)=(i \rho)^{\nu} \exp (i \rho x)[1], \quad \nu=0,1, \quad|\rho| \rightarrow \infty, \quad \rho \in \Omega_{\delta} .
$$

Similarly, we obtain

$$
S^{(\nu)}(x, \lambda)=-\frac{(-i \rho)^{\nu}}{2 i \rho} \exp (-i \rho x)[1], \quad \nu=0,1, \quad|\rho| \rightarrow \infty, \quad \rho \in \Omega_{\delta},
$$

for each fixed $x \in\left(0, b_{1}\right]$.

\section{SOLUTION OF THE INVERSE PROBLEM}

Let the numbers $q\left(b_{2}\right), \ldots, q\left(b_{N-1}\right)$ be known a priori. The inverse problem is formulate as follows.

Inverse problem 1. Given two spectra $\left\{\lambda_{n j}\right\}_{n \geqslant 0}, j=0,1$, construct $q$ on $T$.

In order to solve this inverse problem we will use the ideas of the method of spectral mappings [6]. Let us prove the uniqueness theorem for the solution of Inverse problem 1. For this purpose together with $L_{j}$ we consider boundary value problems $\tilde{L}_{j}$ of the same form but with the other potential $\tilde{q}$. We agree that if a certain symbol $\theta$ denotes an object related to $L_{j}$, then $\tilde{\theta}$ will denote an analogous object related to $\tilde{L}_{j}$.

Theorem 1. If $\lambda_{n j}=\tilde{\lambda}_{n j}, n \geqslant 0, j=0,1$, then $q=\tilde{q}$ on $T$. Thus, the specification of two spectra $\left\{\lambda_{n j}\right\}_{n \geqslant 0}, j=0,1$ uniquely determines the potential $q$. 
Proof. Using knowledge of two spectra $\left\{\lambda_{n j}\right\}_{n \geqslant 0}, j=0,1$, we construct the Weyltype function $M(\lambda)$ via (11) and (12). In particular this yields $M(\lambda) \equiv \tilde{M}(\lambda)$.

For $x \in\left(0, b_{1}\right)$, we consider the functions

$P_{1}(x, \lambda)=\Phi(x, \lambda) \tilde{S}^{\prime}(x, \lambda)-\tilde{\Phi}^{\prime}(x, \lambda) S(x, \lambda), \quad P_{2}(x, \lambda)=\tilde{\Phi}(x, \lambda) S(x, \lambda)-\Phi(x, \lambda) \tilde{S}(x, \lambda)$.

It follows from (18)-(19)that for each fixed $x \in\left(0, b_{1}\right)$,

$$
P_{1}(x, \lambda)=1+O\left(\rho^{-1}\right), \quad P_{2}(x, \lambda)=O\left(\rho^{-1}\right), \quad|\rho| \rightarrow \infty, \quad \rho \in \Omega_{\delta} .
$$

On the other hand, using (10) and the relation $M(\lambda) \equiv \tilde{M}(\lambda)$, we get

$P_{1}(x, \lambda)=C(x, \lambda) \tilde{S}^{\prime}(x, \lambda)-\tilde{C}^{\prime}(x, \lambda) S(x, \lambda), \quad P_{2}(x, \lambda)=\tilde{C}(x, \lambda) S(x, \lambda)-C(x, \lambda) \tilde{S}(x, \lambda)$,

and consequently, for each fixed $x \in\left(0, b_{1}\right)$, the functions $P_{1}(x, \lambda)$ and $P_{2}(x, \lambda)$ are entire in $\lambda$ of order $1 / 2$. Together with $(20)$ this yields $P_{1}(x, \lambda) \equiv 1$ and $P_{2}(x, \lambda) \equiv 0$. Since $\Phi(x, \lambda) S^{\prime}(x, \lambda)-\Phi^{\prime}(x, \lambda) S(x, \lambda) \equiv 1$, it follows that

$$
\begin{gathered}
P_{1}(x, \lambda) \tilde{\Phi}(x, \lambda)+P_{2}(x, \lambda) \tilde{\Phi}^{\prime}(x, \lambda)=\Phi(x, \lambda), \\
P_{1}(x, \lambda) \tilde{S}(x, \lambda)+P_{2}(x, \lambda) \tilde{S}^{\prime}(x, \lambda)=S(x, \lambda) .
\end{gathered}
$$

Therefore,

$$
\Phi(x, \lambda) \equiv \tilde{\Phi}(x, \lambda),
$$

and consequently, $q(x)=\tilde{q}(x)$ for $x \in\left[0, b_{1}\right]$. Using the method of spectral mappings [6] we also obtain an algorithm for constructing the potential $q(x)$ for $x \in\left[0, b_{1}\right]$.

Denote

$$
\Phi_{1}(x, \lambda):=\frac{\Phi(x, \lambda)}{\Phi\left(a_{N}, \lambda\right)}, \quad M_{1}(\lambda):=\Phi_{1}^{\prime}\left(a_{N}, \lambda\right)=\frac{\Phi^{\prime}\left(a_{N}, \lambda\right)}{\Phi\left(a_{N}, \lambda\right)} .
$$

Since $\Phi_{1}\left(a_{N}, \lambda\right)=1, \Phi_{1}\left(b_{N}, \lambda\right)=0$, it follows that the function $M_{1}(\lambda)$ is the Weyl function for equation (4) on the segment $\left[a_{N}, b_{N}\right]$. Taking (15), (21) and (22) into account, we infer $M_{1}(\lambda)=\tilde{M}_{1}(\lambda)$. The specification of the Weyl function $M_{1}(\lambda)$ uniquely determines the potential $q(x)$ for $x \in\left[a_{N}, b_{N}\right]$. This means that Theorem 1 is proved, and the solution of Inverse problem 1 can be found by the following algorithm.

Algorithm. Let two spectra $\left\{\lambda_{n j}\right\}_{n \geqslant 0} j=0,1$, be given.

1. Calculate the Weyl-type function $M(\lambda)$ via (12) and (11).

2. Construct $q(x)$ and $\Phi(x, \lambda)$ for $x \in\left[a_{1}, b_{1}\right]$ using (18)-(20) and the method of spectral mappings.

3. Find $\Phi\left(a_{N}, \lambda\right)$ and $\Phi^{\prime}\left(a_{N}, \lambda\right)$ via $(15)$.

4. Calculate $M_{1}(\lambda)$ by $(22)$.

5. Construct $q(x)$ and $\Phi(x, \lambda)$ for $x \in\left[a_{N}, b_{N}\right]$ by the method of spectral mappings.

Acknowledgements: This work was supported in part by the Russian Ministry of Education and Science (project No. 1.1660.2017/4.6) and by the Russian Foundation for Basic Research (project No. 19-01-00102).

\section{References}

1. Bohner M., Peterson A. Dynamic Equations on Time Scales. Boston, MA, Birkhäuser, 2001. 358 p.

2. Bohner M., Peterson A. Advances in Dynamic Equations on Time Scales. Boston, MA, Birkhäuser, 2003. 348 p. 
3. Marchenko V. A. Sturm-Liouville Operators and Applications. Basel, Birkhäuser, 1986. $393 \mathrm{p}$.

4. Levitan B. M. Inverse Sturm-Liouville Problems. Utrecht, VNU Science Press, 1987. $246 \mathrm{p}$.

5. Freiling G., Yurko V. A. Inverse Sturm-Liouville Problems and Their Applications. New York, NOVA Science Publ., 2001. 305 p.

6. Yurko V. A. Method of Spectral Mappings in the Inverse Problem Theory. Inverse and Ill-Posed Problems Series 31. Utrecht, VSP, 2002. 306 p. DOI: https://doi.org/10.1515/9783110940961

7. McLaughlin J. R. Analytical methods for recovering coefficients in differential equations from spectral data. SIAM Rev., 1986, vol. 28, pp. 53-72.

8. Poeschel J., Trubowitz E. Inverse Spectral Theory. Academic Press, 1987. 192 p.

9. Beals R., Deift P., Tomei C. Direct and inverse scattering on the line. Mathematical Surveys and Monographs. Vol. 28. Providence, RI, AMS, 1988. 209 p.

10. Romanov V. G. Inverse Problems in Mathematical Physics. Utrecht, VNU Science Press, 1987. 367 p.

11. Sakhnovich L. A. Spectral Theory of Canonical Differential Systems. Method of Operator Identities. Operator Theory: Advances and Applications. Vol. 107. Basel, Birkhäuser, 1999. 408 p. DOI: https://doi.org/10.1007/978-3-0348-8713-7

12. Yurko V. A. Inverse spectral problems for Sturm-Liouville operators on graphs. Inverse Problems, 2005, vol. 21, no. 3, pp. 1075-1086. DOI: https://doi.org/10.1088/0266$5611 / 21 / 3 / 017$

13. Arov D., Dym H. Bitangential direct and inverse problems for systems of integral and differential equations. Encyclopedia of Mathematics and its Applications. Vol. 145. Cambridge University Press, 2012. 472 p. DOI: https://doi.org/10.1017/CBO9781139093514

14. Pivovarchik V., Moeller M. Spectral Theory of Operator Pencils, Hermite-Biehler Functions and their Applications. Operator Theory: Advances and Applications. Vol. 246. Cham, Birkhaüser, 2015. 412 p. DOI: https://doi.org/10.1007/978-3-319-17070-1

15. Yurko V. A. Inverse spectral problems for differential operators on spatial networks. Russian Mathematical Surveys, 2016, vol. 71, no. 3, pp. 539-584. DOI: http://dx.doi.org/10.1070/RM9709

Cite this article as:

Yurko V. A. On Recovering Differential Operators on a Closed Set from Spectra. Izv. Saratov Univ. (N. S.), Ser. Math. Mech. Inform., 2019, vol. 19, iss. 4, pp. 389-396. DOI: https://doi.org/10.18500/1816-9791-2019-19-4-389-396

\section{УДК 517.984}

\section{О восстановлении дифрференциальных операторов на замкнутом множестве по спектрам}

\section{В. А. Юрко}

Юрко Вячеслав Анатольевич, доктор фризико-математических наук, заведующий кафедрой математической фризики и вычислительной математики, Саратовский национальный исследовательский государственный университет имени Н. Г. Чернышевского, Россия, 410012, г. Саратов, ул. Астраханская, д. 83, YurkoVA@info.sgu.ru

Рассматриваются дифрференциальные операторы Штурма-Лиувилля на замкнутых множествах вещественной оси. Получены свойства их спектральных характеристик и исследуется обратная задача восстановления операторов по их спектрам. Разработан алгоритм решения обратной задачи и установлена единственность ее решения. Постанов- 
ка и исследование обратных задач существенно зависят от структуры замкнутого множества. Рассматривается важный класс замкнутых множеств, когда множество является объединением конечного набора отрезков и изолированных точек. Для того, чтобы решить обратную задачу для этого класса замкнутых множеств, дается развитие идей метода спектральных отображений. Также установлены и используются связи между функциями типа Вейля, относящиеся к разным подмножествам основного замкнутого множества. C помощью этих идей и свойств получена глобальная конструктивная процедура решения рассматриваемой нелинейной обратной задачи, а также установлена единственность решения этой обратной задачи.

Ключевые слова: диффреренциальные операторы, замкнутые множества, обратная спектральная задача.

Поступила в редакцию: 01.03.2019 / Принята: 28.04.2019 / Опубликована: 02.12.2019

Статья опубликована на условиях лицензии Creative Commons Attribution License (CC-BY 4.0)

Благодарности. Работа выполнена при финансовой поддержке Минобрнауки РФ (проект № 1.1660.2017/4.6) и РФФИ (проект № 19-01-00102).

\section{Образец для цитирования:}

Yurko V. A. On Recovering Differential Operators on a Closed Set from Spectra [Юрко B. A. О восстановлении дифференциальных операторов на замкнутом множестве по спектрам] // Изв. Сарат. ун-та. Нов. сер. Сер. Математика. Механика. Информатика. 2019. Т. 19, вып. 4. C. 389-396. DOI: https://doi.org/10.18500/1816-9791-2019-19-4-389-396 